\title{
Simplified modeling of the human body exposed to power substation electric field using boundary element analysis
}

\author{
D. Poljak ${ }^{1}$, N. Kovač ${ }^{1}$, S. Kraljević ${ }^{2} \&$ C. A. Brebbia ${ }^{3}$ \\ ${ }^{1}$ University of Split, Croatia \\ ${ }^{2}$ HEP Elektrodalmacija, Croatia \\ ${ }^{3}$ Wessex Institute of Technology, UK
}

\begin{abstract}
This paper deals with human exposure to extremely low frequency (ELF) electric fields generated by a transformer substation. The problem is twofold, i.e. it implies the assessment of the power substation electric field and the related current density induced inside the human body. The ELF electric field generated from a power substation is determined by solving the Scalar Potential Integral Equation (SPIE) using the Source Element Method (SEM), a variant of the Indirect Boundary Element Method (IBEM). Knowing the electric field in the vicinity of a substation the current density induced inside a simplified cylindrical model of the human body is obtained by solving the Pocklington integrodifferential equation using the Galerkin-Bubnov Indirect Boundary Element Method (GB-IBEM).
\end{abstract}

\section{Introduction}

Human exposure to extremely low frequency (ELF) fields generated by power substations initiated an increasing public concern regarding possible adverse health effects. A lot of controversy has been caused due to the possible link between the low frequency fields and leukaemia, or certain forms of tumour (e.g. nervous tissue tumour) [1-3] in humans. As the displacement currents at extremely low frequencies are negligible the electric and magnetic fields can be analyzed separately. In the case of the magnetic field exposure the internal currents form close loops, while in the electric field exposure the currents induced in the body have the axial character. 
This paper deals with the assessment of human exposure to ELF electric fields generated by power substations using the indirect boundary element approach, i.e. the method of sources. First, the spatial distribution of the electric field is assessed by solving the Scalar Potential Integral Equation (SPIE) using the source integration procedure known as the Charge Simulation Method (CSM), or the Source Element Method (SEM) [4]. This approach can be also referred to as a variant of the Indirect Boundary Element Method (IBEM).

Second, the current density induced in the human body, as a main parameter for the estimation of low frequency exposure effects proposed by ICNIRP basic restrictions [5], has to be determined. The assessment of the current density induced in the human body due to ELF exposures, mostly to power lines, has already been reported by some researchers having used either analytical $[1,2]$, or numerical techniques [6-10].

The simplified cylindrical representation of the human body, already proved to be efficient in the assessment of human exposure to power line electric fields $[3,10]$, is used in this work for the power station case study. It is worth noting that the main feature of the model is efficiency and rapid estimation of the phenomenon.

\section{Calculation of electric field}

Electric scalar potential at an arbitrary point $P(x, z)$ due to a conductor segment carrying linear charge density, as shown in Figure 1, is defined as [4]:

$$
\varphi(x, z)=\frac{1}{4 \pi \varepsilon} \int_{-L}^{+L} \frac{\rho_{l}\left(x^{\prime}\right) d x^{\prime}}{R}
$$

where: $\rho_{l}$ denotes the line charge density, $2 L$ stands for the wire length, $R$ is the distance between a point on the straight wire and an arbitrary point $P$.

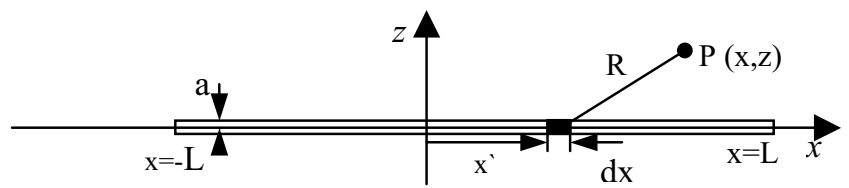

Figure 1: $\quad$ Straight wire geometry.

When the potential $\varphi_{l}$ along the straight wire is known, the integral equation (1) becomes:

$$
\varphi_{l}=\frac{1}{4 \pi \varepsilon} \int_{-L}^{+L} \frac{\rho_{l}\left(x^{\prime}\right) d x^{\prime}}{\sqrt{\left(x-x^{\prime}\right)^{2}+a^{2}}},
$$

where $a$ denotes the wire radius. 
Having performed a discretization of the substation conductors a system of equations for unknown charges along the each segment is obtained.

The integral equation (2) then transforms into a corresponding matrix equation [10]:

$$
\left[\begin{array}{cccccc}
P_{11} & P_{12} & \cdot & \cdot & \cdot & P_{1 n} \\
P_{21} & P_{22} & \cdot & \cdot & \cdot & P_{2 n} \\
\cdot & \cdot & & & & \\
\cdot & \cdot & & & & \\
\cdot & \cdot & & & & \\
P_{n 1} & P_{n 2} & \cdot & \cdot & \cdot & P_{n n}
\end{array}\right] \cdot\left[\begin{array}{c}
q_{1} \\
q_{2} \\
\cdot \\
\cdot \\
\cdot \\
q_{n}
\end{array}\right]=\left[\begin{array}{c}
\varphi_{1} \\
\varphi_{2} \\
\cdot \\
\cdot \\
\cdot \\
\varphi_{n}
\end{array}\right]
$$

where: $\varphi_{1}, \varphi_{2}, \ldots, \varphi_{n}$ stand for the boundary element potentials, $q_{1}, q_{2}, \ldots, q_{n}$ denote the boundary element charges, and $P_{11}, P_{12}, \ldots, P_{n n}$ are Maxwell coefficients.

The unknown charges are obtained by inverting the Maxwell coefficient matrix (3), while the details pertaining to the Maxwell coefficients can be found in [11].

The electric field components in rectangular coordinates at an arbitrary point $(x, y, z)$, generated by an $i$-th boundary element, Figure 2 , are given by [5]:

$$
\begin{aligned}
& E_{x}=-\frac{\partial \varphi}{\partial x}=\frac{q_{i}}{4 \pi \varepsilon_{0} L_{i}}\left[\frac{1}{\sqrt{\left(L_{i}-x\right)^{2}+W^{2}}}-\frac{1}{\sqrt{x^{2}+W^{2}}}\right] \\
& E_{y}=-\frac{\partial \varphi}{\partial y}=\frac{q_{i}}{4 \pi \varepsilon_{0} L_{i}} \frac{y}{W^{2}}\left[\frac{L_{i}-x}{\sqrt{\left(L_{i}-x\right)^{2}+W^{2}}}+\frac{x}{\sqrt{x^{2}+W^{2}}}\right] \\
& E_{z}=-\frac{\partial \varphi}{\partial z}=\frac{q_{i}}{4 \pi \varepsilon_{0} L_{i}} \frac{z}{W^{2}}\left[\frac{L_{i}-x}{\sqrt{\left(L_{i}-x\right)^{2}+W^{2}}}+\frac{x}{\sqrt{x^{2}+W^{2}}}\right]
\end{aligned}
$$

where: $q_{i}$ denotes the charge of $i$-th segment, $L_{i}$ stands for the length of $i$-th segment, and

$$
W^{2}=y^{2}+z^{2} .
$$

As it is visible from Fig 2 the total field components are assembled from each boundary element. 


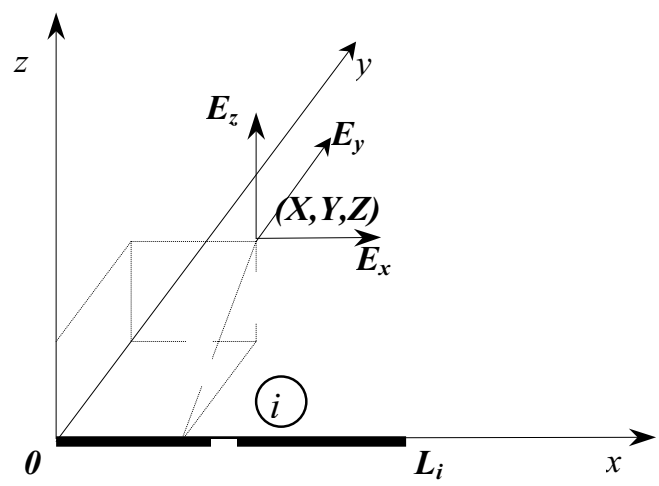

Figure 2: $\quad$ Electric field components produced by $i$-th segment.

\section{Cylindrical antenna model of the human body}

The human body vertically standing on the earth and exposed to the extremely low (ELF) electric field can be represented by a cylindrical antenna model with a uniform cross section and conductivity, i.e. the conducting cylinder of the full length $L$ and radius $a$, as it is shown in Fig. 3.

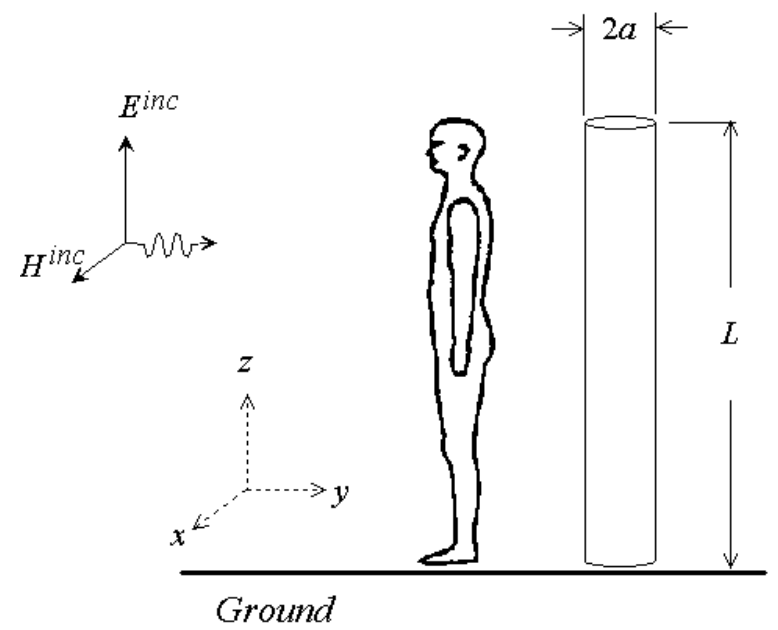

Figure 3: The equivalent antenna model of the human body.

The determination of the current distribution within the human body is based on the solution of the frequency domain Pocklington integro-differential equation. The loaded thick-wire model of the human body exposed to the ELF fields based on the Pocklington equation, which is used in this work, has been 
proposed in [3] and [9]. This equation can be derived starting from the time harmonic Maxwell's equations. The Pocklington integro-differential equation which determines the current along a thick wire is given by [3,9]:

$$
E_{z}^{\text {inc }}=-\frac{1}{4 j \pi \omega \varepsilon_{0}} \int_{-L}^{L}\left[\frac{\partial^{2}}{\partial z^{2}}+k^{2}\right] g_{E}\left(z, z^{\prime}\right) I\left(z^{\prime}\right) d z^{\prime}+Z_{L}(z) I(z)
$$

where $E_{z}^{i n c}$ is the corresponding incident field, $I(z)$ is the axial current distribution $Z_{L}(z)$ is the impedance per unit length of the cylinder, and $g_{E}\left(z, z^{\prime}\right)$ is the kernel of the integral equation which is given by $[3,9]$ :

$$
g_{E}\left(z, z^{\prime}\right)=\frac{1}{2 \pi} \int_{0}^{2 \pi} \frac{e^{-j k R}}{R} d \phi
$$

in which $R$ denotes the distance from the source point $z$ to the observation point $z$, where both are located on the thick wire antenna surface, therefore:

$$
R=\sqrt{\left(z-z^{\prime}\right)^{2}+4 a^{2} \sin ^{2} \frac{\phi}{2}}
$$

The electric properties of the body are taken into account by the character of the impedance $Z_{L}$ which is, for ELF exposures given by:

$$
Z_{L}(z)=\frac{1}{a^{2} \pi \sigma}+Z_{c}
$$

where the capacitive impedance $Z_{c}=-j / \omega \mathrm{C}$ between the antenna and its image accounts for the isolating effect of shoes being worn.

The maximum current will flow from the body into the ground when it is assumed that the person is standing bare footed on a good conducting ground, $Z_{c}=0$, which is assumed in this work.

The induced current density is simply given by:

$$
J_{z}(z)=\frac{I_{z}(z)}{a^{2} \pi}
$$

The Pocklington integro-differential equation for the single thick wire is solved by the Galerkin-Bubnov Indirect Boundary Element Method (GB-IBEM). The details are available elsewhere, e.g. in [3]. 


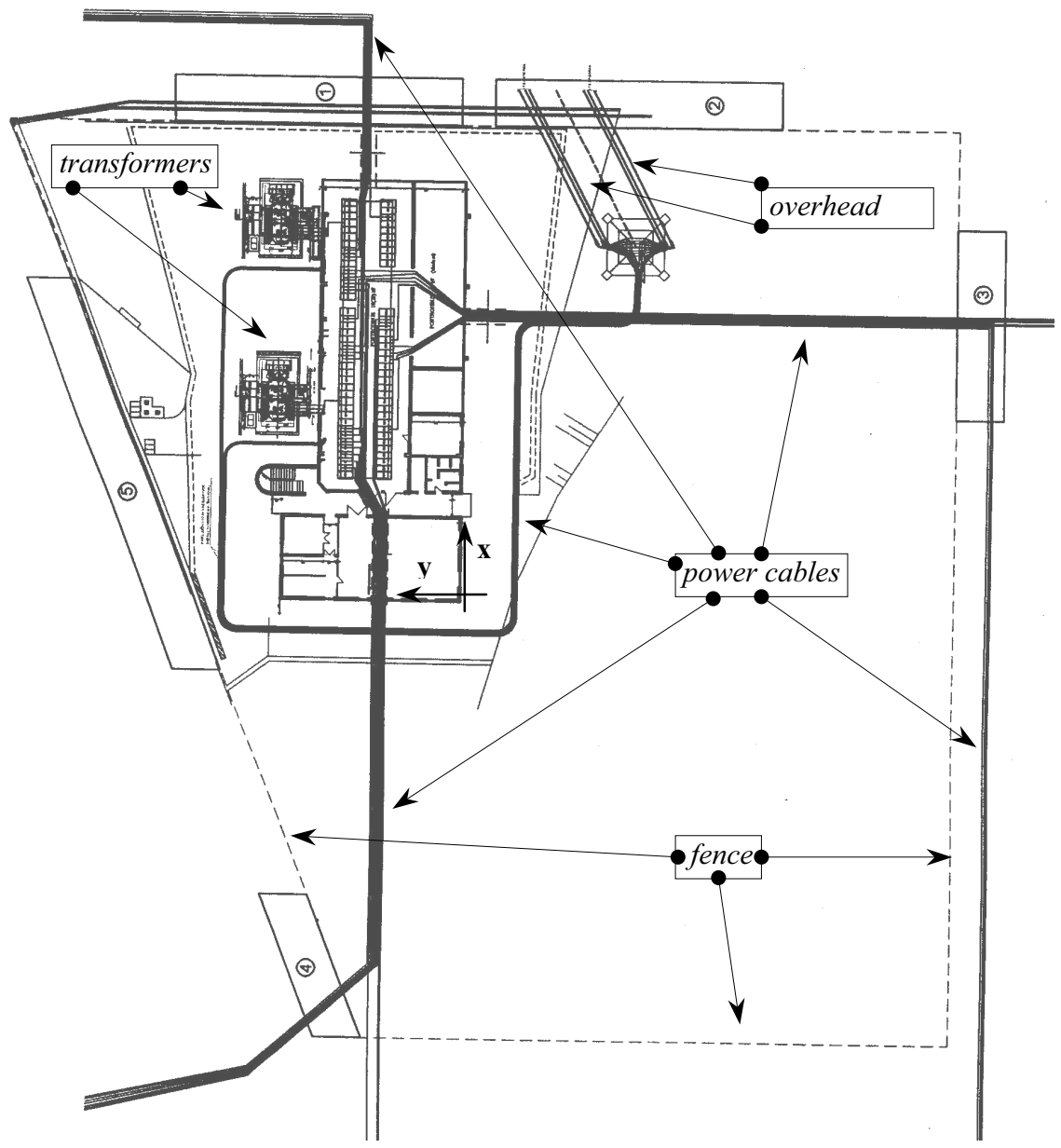

Figure 4: $\quad$ Substation layout.

\section{Computational example}

A computational example is related to the $110 / 10 \mathrm{kV} / \mathrm{kV}$ transmission substation of GIS (Gas-Insulated Substation) type in Split, Croatia which simplified twodimensional layout of the substation is shown in Fig 4. The 50Hz-electric field is calculated at height $z=1 \mathrm{~m}$ above ground. There is no real possibility of a public exposure within the transmission substation, and a professional exposure is strictly limited to duration, thus the calculations are undertaken outside the fence of the substation.

The equipment with grounded shields (power cables), sheaths (GIS buses), or metallic casings (transformers, switchgears) are neglected due to the shielding effect [12], i.e. the metallic enclosures are connected to ground, thus producing 
negligible electric field. Consequently, overhead transmission lines and unshielded conductors are considered for this particular substation. The calculation domains 1 to 5 , in which the higher field values are expected, are assigned in Figure 4. The spatial distribution of electric field over the domain 2, where the highest field value is captured, is shown in Figure 5. It is worth noting that due to the shortness of the unshielded conductors, as well as their considerable distances from the substation fence, the main electric field sources are the overhead lines.

This can be observed in Fig 5 where the maximal electric field is $E_{\max }=380.70$ $\mathrm{V} / \mathrm{m}$. The point of maximum of electric field is located just below the overhead line route.

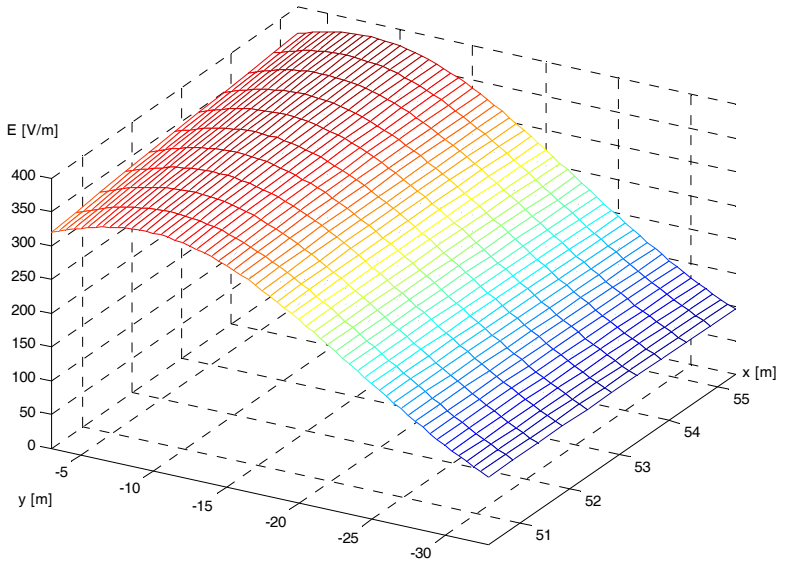

Figure 5: $\quad$ Spatial distribution of the electric field over the domain No. 2.

Once the external electric field is known the current density induced inside the human body can be computed. The human body consists of the various tissues and organs with varying electrical properties such as conductivity $\sigma$ and relative permittivity $\varepsilon_{\mathrm{r}}$. The cylinder length is $L=1.75 \mathrm{~m}$, radius is $a=0.14 \mathrm{~m}$, while the average conductivity of the body is approximately $\sigma \approx 0.5 \mathrm{~S} / \mathrm{m}$.

The induced current densities and axial currents calculated for the case of maximal field values are presented in Table 1 .

Table 1: $\quad$ Maximal field values and related internal current densities.

\begin{tabular}{|c|c|c|c|}
\hline Domain & $E(\mathrm{~V} / \mathrm{m})$ & $J\left(\mu \mathrm{A} / \mathrm{m}^{2}\right)$ & $I_{z}(\mu \mathrm{A})$ \\
\hline 1 & 327.546 & 85.31 & 5.253 \\
\hline 2 & 380.704 & 99.15 & 6.105 \\
\hline 3 & 31.9 & 8.31 & 0.512 \\
\hline
\end{tabular}


In addition, it is evident that the maximal value of the computed field in domain $2(380.704 \mathrm{kV} / \mathrm{m})$ is appreciably lower from the exposure limits for both occupational $(10 \mathrm{kV} / \mathrm{m})$ and general population $(5 \mathrm{kV} / \mathrm{m})$, respectively proposed by ICNIRP guidelines [5].

In addition, the maximal value of internal current density $0.09915 \mathrm{~mA} / \mathrm{m}^{2}$ compared to the basic restrictions proposed by ICNIRP [5] is also far below exposure limits for both occupational $\left(10 \mathrm{~mA} / \mathrm{m}^{2}\right)$ and general population $\left(2 \mathrm{~mA} / \mathrm{m}^{2}\right)$, respectively.

The longitudinal distribution of the induced current density in the body model, for different values of the external field, is shown in Fig 6.

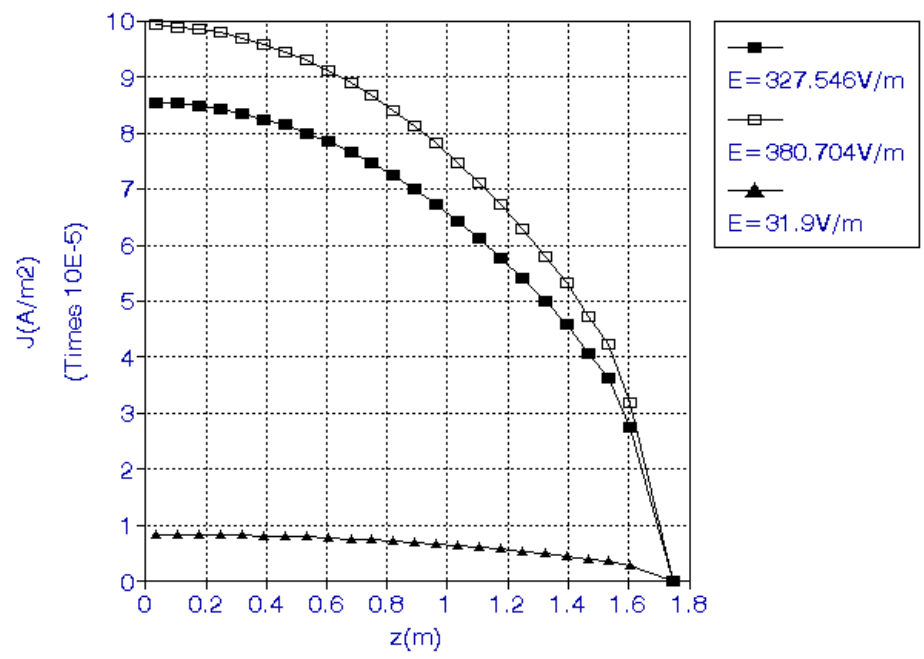

Figure 6: Current density distribution along the cylindrical body.

\section{Concluding remarks}

Human exposure to ELF electric fields generated from power substations is analyzed in this paper using the cylindrical model of the human body. The problem is twofold involving the calculation of power station electric field and the related internal current density, respectively. The electric field generated by the power substation is calculated by the Source Element Method (SEM) representing a variant of Indirect Boundary Element Method (IBEM). The current density induced inside the human body is obtained by solving the corresponding Pocklington integro-differential equation via the Galerkin-Bubnov Indirect Boundary Element Method (GB-IBEM). Obtained computational results for the electric field and the internal current density, respectively, are appreciably below the reference levels and basic restrictions, respectively, proposed by the ICNIRP guidelines. 


\section{References}

[1] King, R.W.P., Sandler, S.S., Electric Fields and Currents Induced in Organs of the Human body When Exposed to ELF and VLF Electromagnetic Fields, Radio Sci., Vol. 31, pp. 1153-1167, Sept.-Oct. 1996.

[2] King, R.W.P., Fields and Currents in the Organs of the Human Body When Exposed to Power Lines and VLF Transmitters, IEEE Trans. Biomedical Eng., Vol. 45, No 4, pp. 520-530, April 1998.

[3] D. Poljak: Human Exposure to Electromagnetic Fields, WIT Pres, Southampton-Boston, 2003.

[4] D. Poljak, C. A. Brebbia: Boundary Elements for Electrical Engineers, WIT Press, Southampton-Boston, 2005.

[5] ICNIRP Guidelines for Limiting Exposure to Time-Varying, Electric, Magnetic and Electromagnetic Fields (up to $300 \mathrm{GHz}$ ), Health Phys., Vol. 74, 4 (1998), 494-522

[6] T.W. Dawson, K. Caputa, M.A. Stuchly, High-Resolution Organ Dosimetry for Human Exposure to Low Frequency Electric Fields, IEEE Trans. Power Delivery, Vol. 13, No 2, pp. 366-373, April 1998.

[7] Chiba, A., Isaka, K., Yokoi Y., Nagata M., Kitagav M., Matsuo T., Application of Finite Element Method to Analysis of Induced Current Densities Inside Human Model Exposed to $60 \mathrm{~Hz}$ Electric Field, IEEE Trans. Power Apparatus and Systems, Vol. PAS-103, No. 7, pp 18951901, July 1984.

[8] Gandhi, O.P., Chen, J.Y., Numerical Dosimetry at Power Line Frequencies Using Anatomically Based Models, Bioelectromagnetics Suppl., Vol. 1, pp. 43-60, 1992.

[9] Poljak, D., Rashed, Y., The Boundary Element Modelling of the Human Body exposed to the ELF Electromagnetic Fields, Engineering Analysis with Boundary Elements, 26, pp 871-875, 2002.

[10] D. Poljak, A. Peratta, C.A. Brebbia, A 3D BEM Modeling of Human Exposure to ELF Electric Fields, BEM XVII, Incorporating Electrical Engineering and Electromagnetics, pp. 441-451, Orlando, USA, March 2005.

[11] J.E.T. Villas, F.C. Maia, D. Mukhedkar, V.S. Da Costa: Computation of Electric Fields Using Ground Grid Performance Equations, IEEE Trans. on Power Delivery, 2(3), pp. 709-716, July 1987.

[12] S. A. Sebo, R. Caldecott: Scale Model Studies Of AC Substation Electric Fields, IEEE Trans. on Power Apparatus and Systems, 98(3), pp. 926-939, May/June 1979. 